\title{
Axisymmetric dynamo action is possible with anisotropic conductivity
}

\author{
Franck Plunian $\oplus^{1, *}$ and Thierry Alboussière $\oplus^{2, \dagger}$ \\ ${ }^{1}$ Université Grenoble Alpes, Université Savoie Mont Blanc, CNRS, IRD, IFSTTAR, ISTerre, 38000 Grenoble, France \\ ${ }^{2}$ Univ. Lyon, UCBL, ENS de Lyon, CNRS, UMR 5276 LGL-TPE, F-69622 Lyon, France
}

(Received 24 June 2019; revised manuscript received 4 October 2019; accepted 14 February 2020; published 16 March 2020)

\begin{abstract}
A milestone of dynamo theory is Cowling's theorem, known in its modern form as the impossibility for an axisymmetric velocity field to generate an axisymmetric magnetic field by dynamo action. Using anisotropic electrical conductivity, we show that an axisymmetric dynamo is in fact possible with a motion as simple as solid-body rotation. On top of that, the instability analysis can be conducted entirely analytically, leading to an explicit expression of the dynamo threshold, which is the only example in dynamo theory.
\end{abstract}

DOI: 10.1103/PhysRevResearch.2.013321

\section{INTRODUCTION}

Since the pioneering study of Cowling [1] there has been a constant effort to improve the demonstration of the so-called Cowling's (antidynamo) theorem. In its modern form this theorem states that an axisymmetric magnetic field cannot be generated by dynamo action under the assumption of axisymmetry of velocity field, electrical conductivity, magnetic permeability, and shape of the conductor [2-6]. Cowling's theorem encompasses time-dependent flows $[7,8]$, nonsolenoidal flows, and variable conductivity $[9,10]$. However, nothing has yet been said about the effect of an anisotropic electrical conductivity and how in this case Cowling's theorem is overcome. A demonstration of dynamo action with shear and anisotropic conductivity has already been given [11] but for a different geometry and within asymptotic limits relevant to the fast dynamo problem.

Beyond its theoretical interest, this issue is relevant to at least three fields of physics. In astrophysics it is well known that in the mean-field approximation, an anisotropic tensor of magnetic diffusivity may naturally occur from anisotropic gradients of magnetohydrodynamic turbulence [12]. In plasma physics, just like thermal conductivity [13], the electrical conductivity in the magnetic field direction is different from the electrical conductivity in the direction perpendicular to the magnetic field [14]. This usually occurs in a plasma which is already magnetized. Although this does not preclude dynamo action, we will not examine this issue here, considering that there is no external magnetic field. Finally, as will be shown below, a dynamo experiment can be designed on the basis of our anisotropic conductivity model.

\footnotetext{
*Franck.Plunian@univ-grenoble-alpes.fr

${ }^{\dagger}$ Thierry.Alboussiere@ens-lyon.fr

Published by the American Physical Society under the terms of the Creative Commons Attribution 4.0 International license. Further distribution of this work must maintain attribution to the author(s) and the published article's title, journal citation, and DOI.
}

The results show that such an experiment is feasible, which is welcome because experimental dynamo demonstrations are rather rare.

\section{ANISOTROPIC CONDUCTIVITY}

Let us consider a material of electrical conductivity $\sigma$ such that $\sigma=\sigma_{1}$ in a given direction $\mathbf{q}$, and $\sigma=\sigma_{0} \geqslant \sigma_{1}$ in the directions perpendicular to $\mathbf{q}$. We choose $\mathbf{q}$ as a unit vector in the horizontal plane,

$$
\mathbf{q}=\cos \alpha \mathbf{e}_{r}+\sin \alpha \mathbf{e}_{\theta},
$$

where $\left(\mathbf{e}_{r}, \mathbf{e}_{\theta}, \mathbf{e}_{z}\right)$ is a cylindrical coordinate system and $\alpha$ a constant angle. In a companion paper another choice for $\mathbf{q}$, within a Cartesian frame, is studied [15].

In Fig. 1 the curved lines correspond to the directions of the large conductivity $\sigma_{0}$. They are perpendicular to $\mathbf{q}$ and describe logarithmic spirals.

Writing Ohm's law $\mathbf{j}=\sigma_{1} \mathbf{E}$ in the direction of $\mathbf{q}$ and $\mathbf{j}=$ $\sigma_{0} \mathbf{E}$ in the directions perpendicular to $\mathbf{q}$ leads to the following conductivity tensor:

$$
\sigma_{i j}=\sigma_{0} \delta_{i j}+\left(\sigma_{1}-\sigma_{0}\right) q_{i} q_{j}
$$

Inversing (2) leads to the resistivity tensor [11]:

$$
R_{i j}=\frac{1}{\sigma_{0}} \delta_{i j}+\left(\frac{1}{\sigma_{1}}-\frac{1}{\sigma_{0}}\right) q_{i} q_{j} .
$$

We consider the solid-body rotation $\mathbf{u}$ of a cylinder of radius $r_{0}$ embedded in an infinite medium at rest (Fig. 1), both regions having the same resistivity tensor $R_{i j}$.

The magnetic induction $\mathbf{B}$ satisfies the equation

$$
\partial_{t} \mathbf{B}=\nabla \times(\mathbf{u} \times \mathbf{B})-\nabla \times([\eta] \nabla \times \mathbf{B}),
$$

where $[\eta]$ is the magnetic diffusivity tensor defined as $\eta_{i j}=$ $R_{i j} / \mu_{0}, \mu_{0}$ being the magnetic permeability of vacuum. Renormalizing the distance, magnetic diffusivity, and time by $r_{0},\left(\mu_{0} \sigma_{0}\right)^{-1}$ and $\mu_{0} \sigma_{0} r_{0}^{2}$, respectively, the dimensionless form of the induction equation is identical to (4) but with

$$
\eta_{i j}=\delta_{i j}+\eta_{1} q_{i} q_{j}, \quad \eta_{1}=\frac{\sigma_{0}}{\sigma_{1}}-1,
$$



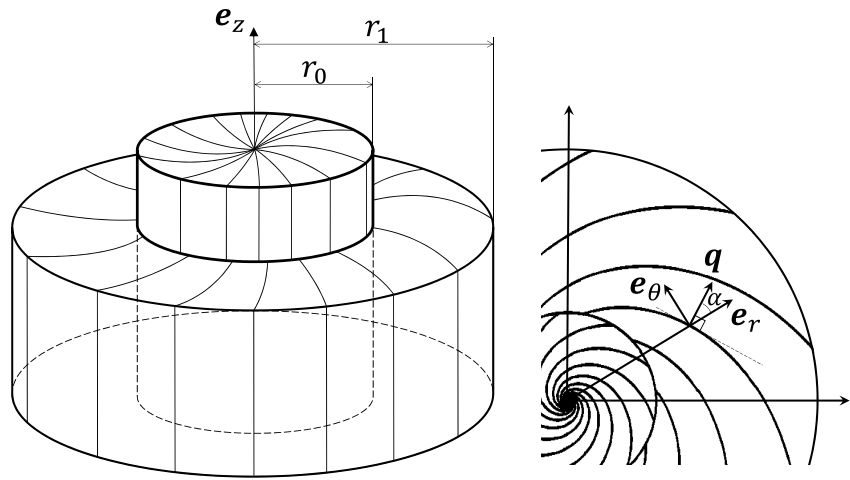

FIG. 1. Left: The inner cylinder of radius $r_{0}$ rotates as a solid body within an outer cylinder at rest. The radial boundary $r_{1}$ of the outer cylinder is rejected at infinity. In the limit $\eta_{1}=\infty$ the electric currents follow logarithmic spiral trajectories. Right: The vector $\mathbf{q}$, which makes a constant angle $\alpha$ with the radial direction, is perpendicular to the spiraling current.

and

$$
\mathbf{u}=\left\{\begin{array}{ll}
r \Omega \mathbf{e}_{\theta}, & r<1 \\
0, & r>1
\end{array},\right.
$$

where $\Omega$ is the dimensionless angular velocity of the inner cylinder.

\section{RESOLUTION}

Provided the velocity is stationary and $z$ independent, an axisymmetric magnetic induction can be searched in the form

$$
\mathbf{B}(r, z, t)=\tilde{B} \mathbf{e}_{\theta}+\nabla \times\left(\tilde{A} \mathbf{e}_{\theta}\right),
$$

with $(\tilde{A}, \tilde{B})=(A, B) \exp (\gamma t+i k z)$, where $\gamma$ is the instability growth rate, $k$ the vertical wave number of the corresponding eigenmode, and where $A$ and $B$ depend only on the radial coordinate $r$. Thus the magnetic induction takes the form

$$
\mathbf{B}=\left(-i k A, B, \frac{1}{r} \partial_{r}(r A)\right) \exp (\gamma t+i k z),
$$

with dynamo action corresponding to $\Re\{\gamma\}>0$.

From Eqs. (6) and (8) we find that $\nabla \times(\mathbf{u} \times \mathbf{B})=0$ in each region $r<1$ and $r>1$. Replacing (5) and (6) in the induction equation (4) leads to

$$
\begin{gathered}
\gamma A+D_{k}(A)=i \eta_{1} c s k B-\eta_{1} s^{2} D_{k}(A), \\
\gamma B+D_{k}(B)=-i \eta_{1} c s k D_{k}(A)-\eta_{1} c^{2} k^{2} B,
\end{gathered}
$$

where $D_{v}(X)=v^{2} X-\partial_{r}\left[\frac{1}{r} \partial_{r}(r X)\right], c=\cos \alpha$ and $s=\sin \alpha$.

Looking for stationary solutions, the dynamo threshold corresponds to $\gamma=0$. Then the system (9)-(10) implies

$$
D_{\tilde{k}}(B)=D_{k}\left(B-i \frac{c k}{s} A\right)=0,
$$

where

$$
\tilde{k}=k\left(\frac{1+\eta_{1}}{1+\eta_{1} s^{2}}\right)^{1 / 2} .
$$

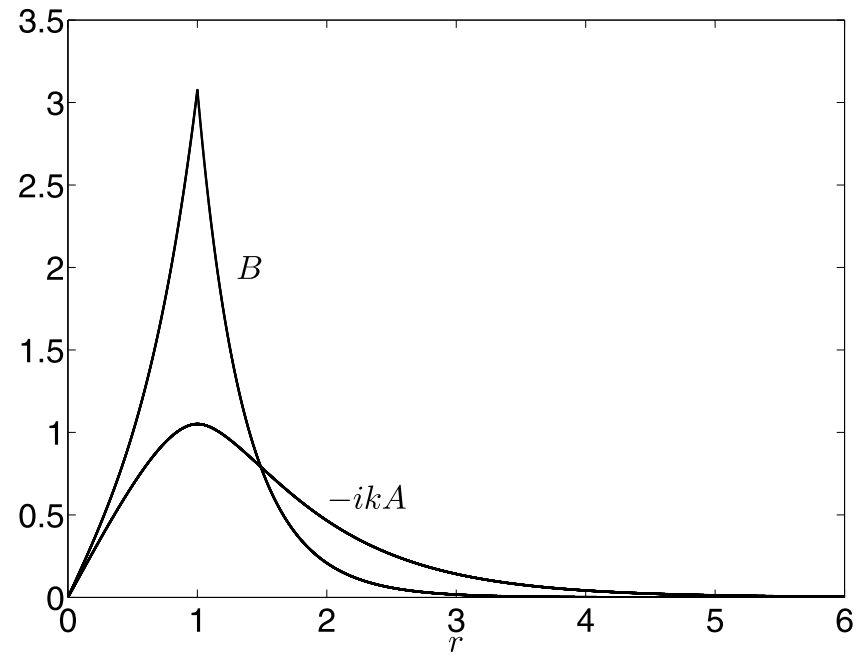

FIG. 2. Eigenmodes $-i k A$ and $B$ vs $r$ for $\eta_{1}=\infty, k=1.1, \alpha=$ $0.16 \pi, \lambda=\Gamma(k)$, and $\mu=-\Gamma(\tilde{k})$.

The solutions of $D_{v}(X)=0$ being a linear combination of $I_{1}(v r)$ and $K_{1}(v r)$, we find

$$
\begin{aligned}
& r<1,\left\{\begin{array}{l}
A=\frac{s}{i c k}\left(\lambda \frac{I_{1}(\tilde{k} r)}{I_{1}(\tilde{k})}+\mu \frac{I_{1}(k r)}{I_{1}(k)}\right) \\
B=\lambda \frac{I_{1}(\tilde{k} r)}{I_{1}(\tilde{k})}
\end{array}\right. \\
& r>1,\left\{\begin{array}{l}
A=\frac{s}{i c k}\left(\lambda \frac{K_{1}(\tilde{k} r)}{K_{1}(\tilde{k})}+\mu \frac{K_{1}(k r)}{K_{1}(k)}\right) \\
B=\lambda \frac{K_{1}(\tilde{k} r)}{K_{1}(\tilde{k})},
\end{array}\right.
\end{aligned}
$$

where $I_{1}$ and $K_{1}$ are modified Bessel functions of first and second kind. In (13) and (14) the following boundary conditions have been applied to $A$ and $B$ : finite values at $r=0$, continuity at $r=1$, and $\lim _{r \rightarrow \infty} A, B=0$.

From (8), the continuity of $\mathbf{B}$ is satisfied provided $\partial_{r} A$ is also continuous at $r=1$. From (13) and (14) this leads to the following identity between $\lambda$ and $\mu$ :

$$
\lambda \Gamma(\tilde{k})+\mu \Gamma(k)=0,
$$

with

$$
\Gamma(x)=x\left(\frac{I_{0}(x)}{I_{1}(x)}+\frac{K_{0}(x)}{K_{1}(x)}\right) \equiv\left[I_{1}(x) K_{1}(x)\right]^{-1},
$$

the last equality coming from the Wronskian relation $I_{m}(x) K_{m+1}(x)+I_{m+1}(x) K_{m}(x)=1 / x$.

In Fig. 2 the eigenmodes $-i k A$ and $B$ are plotted versus $r$ for $\lambda=\Gamma(k)$ and $\mu=-\Gamma(\tilde{k})$ such that (15) is satisfied. Both $-i k A$ and $B$ reach their maximum at $r=1$.

Finally, the tangential components of the electric field $\mathbf{E}=-\mathbf{u} \times \mathbf{B}+[\eta] \nabla \times \mathbf{B}$ have to be continuous at $r=1$. The continuity of $\mathbf{E}_{z}$ implies the following identity:

$$
\left(\partial_{r} B-i k \Omega A\right)\left(r=1^{-}\right)=\partial_{r} B\left(r=1^{+}\right) .
$$

According to Fig. 2, from which we have $-i k A \geqslant 0, \partial_{r} B(r<$ $1)>0$ and $\partial_{r} B(r>1) \leqslant 0$, the only way to satisfy (17) is to have $\Omega<0$. Replacing (13), (14), and (15) in (17) leads to the 


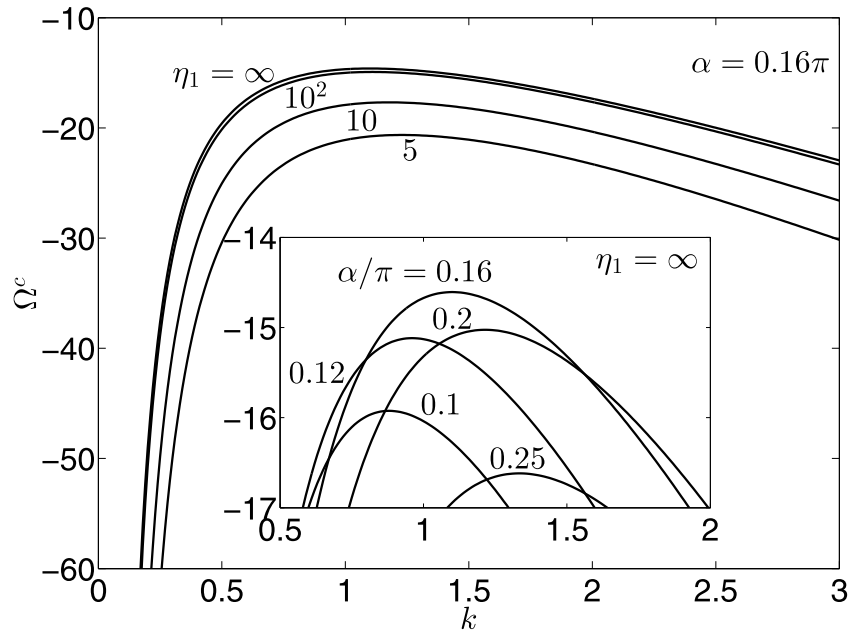

FIG. 3. Curves of the dynamo threshold $\Omega^{c}$ vs $k$ for $\alpha=0.16 \pi$ and $\eta_{1}=5,10,10^{2}, \infty$. Inset: $\eta_{1}=\infty$ and $\alpha / \pi=$ $0.1,0.12,0.16,0.2,0.25$.

dynamo threshold

$$
\Omega^{c}=\frac{c}{s}\left(I_{1}(\tilde{k}) K_{1}(\tilde{k})-I_{1}(k) K_{1}(k)\right)^{-1} .
$$

As previously noted, we find negative values of $\Omega^{c}$, dynamo action corresponding to $|\Omega| \geqslant\left|\Omega^{c}\right|$. In Fig. 3 the curves of the dynamo threshold are plotted for different values of $\eta_{1}$ and $\alpha$. The minimum value of $\left|\Omega^{c}\right|$ is obtained for $\eta_{1} \rightarrow \infty$, $k^{*}=1.1$, and $\alpha^{*}=0.16 \pi$ :

$$
\Omega^{*}=\min _{\eta, k, \alpha}\left|\Omega^{c}\right|=14.61 .
$$

\section{DYNAMO MECHANISM}

The dynamo mechanism can be described as a two-step process as illustrated in Fig. 4. The boundary condition (17) implies that $\mathbf{B}_{\theta}$ is generated from $\mathbf{B}_{r}$ by differential rotation between the inner and outer cylinders. This leads to distortion of magnetic field lines as shown in the right of Fig. 4. In return, the first term on the right-hand side of (9) corresponds to the
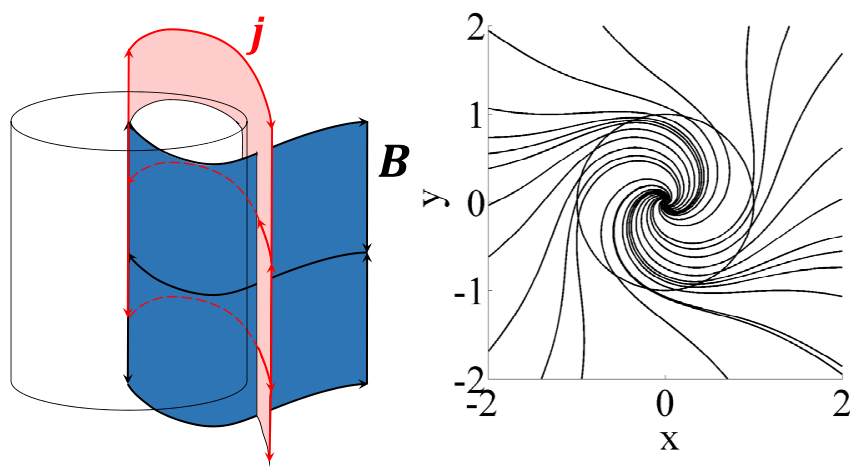

FIG. 4. Left: Three-dimensional sketch of some trajectories of the current density $\mathbf{j}$ and the magnetic field $\mathbf{B}$. Right: Magnetic field lines in the horizontal plane $z=0$. The magnetic field is distorted by the differential rotation while the current density is bent by the conductivity anisotropy. generation of $\mathbf{B}_{r}$ from $\mathbf{B}_{\theta}$, provided $\eta_{1} c s k \neq 0$. This appears more clearly by rewriting (9) and (10) as

$$
\begin{gathered}
\gamma \mathbf{B}_{r}=\eta_{1} c s k^{2} \mathbf{B}_{\theta}-\left(1+\eta_{1} s^{2}\right) D_{k}\left(\mathbf{B}_{r}\right), \\
\gamma \mathbf{B}_{\theta}=\eta_{1} c s D_{k}\left(\mathbf{B}_{r}\right)-\left(D_{k}+\eta_{1} c^{2} k^{2}\right) \mathbf{B}_{\theta} .
\end{gathered}
$$

In the left of Fig. 4 the horizontal currents are represented to follow the direction of logarithmic spirals. To show it, the current density $\boldsymbol{j}=\boldsymbol{\nabla} \times \mathbf{B}$ is written in the form

$$
\mathbf{j}=\left(-i k B, D_{k}(A), \frac{1}{r} \partial_{r}(r B)\right) \exp (\gamma t+i k z) .
$$

From (9) taken at the threshold $\gamma=0$, we find that

$$
j_{\theta}=-\frac{\eta_{1} c s}{1+\eta_{1} s^{2}} j_{r},
$$

corresponding to the equation of logarithmic spirals. In the limit $\eta_{1} \rightarrow \infty$ we find that $\mathbf{j} \cdot \mathbf{q}=0$, the currents following the trajectories given in Fig. 1 .

Dynamo action thus occurs through differential rotation conjugated to anisotropic diffusion. For $\eta_{1}=0$ (isotropic diffusion) or $c s=0$, in Eqs. (20) and (21) $\mathbf{B}_{r}$ and $\mathbf{B}_{\theta}$ are decoupled, canceling any hope of dynamo action in accordance with Cowling's theorem.

It is interesting to note that in (20) and (21), in each equation it is the first term on the right-hand side which supports dynamo action. These terms correspond to the off-diagonal coefficients of the anisotropic diffusivity tensor (5). Therefore the diagonal and off-diagonal coefficients act respectively against and in favor of dynamo action.

\section{CONCLUSIONS}

The neutral point argument of Cowling relies on the impossibility, in an axisymmetric configuration, of maintaining a toroidal current density [1]. This argument fails as soon as the conductivity is a tensor, because in this case, the cross product of a toroidal velocity field with a poloidal magnetic field can actually produce a toroidal current density. In other words, the anisotropic conductivity forces the current density to follow spiraling trajectories with nonzero azimuthal components, thus overcoming Cowling's theorem.

Beyond the fact that with an anisotropic conductivity an axisymmetric dynamo can be operated from a simple solid-body rotation, it is interesting to put some numbers on the previous results. Considering an inner cylinder of radius $r_{0}=0.05 \mathrm{~m}$, taking the conductivity of copper $\mu_{0} \sigma_{0} \approx 72.9 \mathrm{~s} \mathrm{~m}^{-2}$ leads to a dynamo threshold $f^{*}=\Omega^{*}\left(2 \pi \mu_{0} \sigma_{0} r_{0}^{2}\right)^{-1} \approx 12.8 \mathrm{~Hz}$. Provided the cylinder height and outer radius $r_{1}$ are sufficiently large, this is experimentally achievable. Such an anisotropic conductivity can be easily manufactured by alternating thin layers of two materials with different conductivities and a logarithmic spiral arrangement of these thin layers. Of course, the resulting conductivity is no longer homogeneous and, more importantly, it does not satisfy the axisymmetry hypothesis of Cowling's theorem. However, provided the layers are thin enough, an anisotropic conductivity model is relevant to 
design such a dynamo experiment. Another dynamo experiment design with spiraling wires has been studied [16].
Though the geometry is different, the dynamo threshold is comparable to the present one.
[1] T. G. Cowling, Mon. Not. R. Astr. Soc. 94, 39 (1934).

[2] H. K. Moffatt, Magnetic Field Generation in Electrically Conducting Fluids (Cambridge University Press, Cambridge, 1978).

[3] D. J. Ivers and R. W. James, Philos. Trans. Roy. Soc. London, Ser. A 312, 179 (1984).

[4] D. Fearn, P. Roberts, and A. Soward, in Pitman Research Notes in Mathematics Series 168, edited by G. Galdi and B. Straughan (Longman Scientific and Technical, New York, USA, 1988), pp. 60-324.

[5] M. Proctor, in Mathematical Aspects of Natural Dynamos, edited by E. Dormy and A. Soward (Chapman and Hall/CRC, Boca Raton, FL, 2007), pp. 18-41.

[6] R. Kaiser and A. Tilgner, SIAM J. Appl. Math. 74, 571 (2014).

[7] G. Backus, Astrophys. J. 125, 500 (1957).

[8] S. I. Braginskii, Soviet Phys. JETP 20, 1462 (1965).
[9] R. Hide and T. N. Palmer, Geophys. Astrophys. Fluid Dyn. 19, 301 (1982).

[10] D. Lortz, R. Meyer-Spasche, and W. Törnig, Math. Methods Appl. Sci. 4, 91 (1982).

[11] M. S. Ruderman and A. A. Ruzmaikin, Geophys. Astrophys. Fluid Dyn. 28, 77 (1984).

[12] F. Krause and K. H. Rädler, Mean-Field Magnetohydrodynamics and Dynamo Theory (Pergamon Press, Oxford, 1980).

[13] M. Onofri, F. Malara, and P. Veltri, Phys. Rev. Lett. 105, 215006 (2010).

[14] S. I. Braginskii, in Reviews of Plasma Physics, edited by M. A. Leontovich, Vol. 1 (Consultants Bureau, New York, 1965), pp. 205-311.

[15] T. Alboussière, K. Drif, and F. Plunian, Phys. Rev. E 101, 033107 (2020).

[16] J. Priede and R. Avalos-Zúñiga, Phys. Lett. A 377, 2093 (2013). 